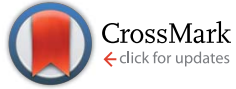

Cite this: RSC Adv., 2015, 5, 107083

Received 18th September 2015 Accepted 27th November 2015

DOI: $10.1039 / \mathrm{c} 5 \mathrm{ra} 19273 \mathrm{k}$

www.rsc.org/advances

\section{A highly porous, light weight 3D sponge like graphene aerogel for electromagnetic interference shielding applications $\uparrow$}

\author{
Sweta Singh, ${ }^{a}$ Prashant Tripathi, ${ }^{a}$ Ashish Bhatnagar, ${ }^{a}$ Ch. Ravi Prakash Patel, ${ }^{a}$ \\ Avanish Pratap Singh, ${ }^{\text {b }}$ S. K. Dhawan, ${ }^{b}$ Bipin Kumar Gupta ${ }^{b}$ and O. N. Srivastava*a
}

Here we report the microwave shielding properties of a light weight three dimensional (3D) sponge like graphene aerogel (GA) derived from graphene oxide (GO). GA is a new exotic form of graphene nanosheet, which shows improved shielding features as compared to its pristine counterpart. The structural and microstructural characteristics of this new indigenous 3D sponge like graphene aerogel architecture have been probed by XRD, Raman, SEM and TEM/HRTEM. Furthermore, the porosity of this newly synthesized structure has been investigated by the Brunauer-Emmett-Teller (BET) method, which confirms the high surface area of $\sim 516 \mathrm{~m}^{2} \mathrm{~g}^{-1}$ with an average pore diameter of $\sim 2.5 \mathrm{~nm}$. The high surface area and better porosity improve the EMI shielding effectiveness of GA. Simultaneously, the GA nanostructure also enhances the dielectric properties which provide a better alternative for EMI shielding materials as compared to GO. This engineered GA exhibits enhanced shielding effectiveness ( $\sim 20.0$ $\mathrm{dB}$ at $0.20 \mathrm{~g}$ in a frequency region of 12.4 to $18.0 \mathrm{GHz}$ ) as compared to the conventional GO. Thus, the result of the EMI shielding of GA offers a new ingenious nanostructure which can be used as an EMI pollutant quencher for next-generation EMI shielding devices.

In the modern era, electromagnetic shielding materials are highly desirable to try to protect the environment from EMI pollutants, which are emanating from most electronic devices like TVs, computers, mobiles, etc. ${ }^{1}$ In the past few years, the conventional wisdom of using EMI shielding materials was based on metal films or plates, but they have their own limitations. These metallic systems are heavy, corrosion prone and have poor processibility. ${ }^{2,3}$ Simultaneously, their shielding effectiveness was mainly based on reflection rather than absorption, which prevents their application in various areas, including in the military and in aircraft. This is since the

${ }^{a}$ Nanoscience Centre, Department of Physics (Centre of Advanced Studies), Banaras Hindu University, Varanasi-221005, India

${ }^{b}$ CSIR - National Physical Laboratory, New Delhi-110 012, India.E-mail: heponsphy@ gmail.com

$\dagger$ Electronic supplementary information (ESI) available: Details of the synthesis of GA and locally fabricated supercritical dryer. See DOI: 10.1039/c5ra19273k reflected electromagnetic waves will be received and detected by an adversary's reconnaissance instrument. Therefore, a continuous search is going on to develop novel materials associated with excellent absorption features for the purpose of reducing electromagnetic interference (EMI) in circuits, chips and radiation controllers. ${ }^{4}$ Recently, immense attempts have been made in the search of high-performance EMI shielding materials with excellent absorption capabilities. In this regard, highperformance EMI shielding materials which are light weight and flexible in nature are highly desirable. This is due to their practical applications in various fields like in aircraft, aerospace, automobiles and fast-growing next-generation flexible electronics such as portable electronics and wearable devices. ${ }^{5}$ Carbon materials such as intercalated graphite, ${ }^{6}$ carbon nanotubes (CNTs), ${ }^{7,8}$ porous carbon, ${ }^{\mathbf{9}, 10}$ carbon nanopowders, ${ }^{\mathbf{1 1}}$ carbon fibres, ${ }^{12}$ graphene ${ }^{\mathbf{1 3 , 1 4}}$ and reduced graphene oxide $(\mathrm{RGO})^{15}$ have been recently reported to absorb electromagnetic waves. Nowadays, two-dimensional layered materials and their composites $^{16-19}$ such as graphene and its derivatives are examined as potential microwave absorbers due to their superior electronic, thermal, and mechanical properties. One of the big advantages of using such nanostructures is their high surface areas. It is also established that carbon-based layered materials provide highly efficient microwave absorbers as compared to nanotubes or nanorod like structures. ${ }^{20}$

In the recent past, significant improvements have been made to the self-assembly of nanomaterials into 3D forms such as hydrogels, aerogels, and some other microporous or mesoporous frameworks. ${ }^{21}$ As a consequence of the $3 \mathrm{D}$ interconnected carbon skeleton, porous carbons provide a tremendous microwave absorption capacity. ${ }^{22-24}$ Compared to microporous foams like the graphene-polymer microcellular foams, ${ }^{25}$ microporous aerogels are advantageous in several ways. Due to the availability of a wide range of fabrication processes and the ability of pore morphologies to be reduced to less than $100 \mathrm{~nm}$, chemically derived GO based graphene aerogels are exotic 3D graphene structures reported in literature. ${ }^{26}$ Intrinsic properties of GA like its microporosity, high electrical 
conductivity and high specific surface area are advantageous in various applications such as in hydrogen storage, catalysis, batteries, filtration, insulation, sorbents and supercapacitors. ${ }^{26}$ GA, with its very high specific surface area, microporosity, very low density and excellent electrical conductivity, is a promising 3D material for high-performance EMI shielding applications. Moreover, the high microporosity of GA gives rise to a high polarity, which leads to a good absorption capacity. This arises due to the enhanced dielectric loss because of the high polarization and presence of defects in the GA nanostructures. These could make GA an outstanding material for next-generation microwave shielding applications.

In this communication, we report a facile method of producing large-scale GA and demonstrate its highly suitable application as an EMI shielding material. The desired microwave shielding properties of the light weight (apparent density $\sim 75 \mathrm{mg} \mathrm{cm}^{-3}$, its method of calculation is given in the ESI†) 3D GA depend on its parent material GO, which is used during the synthesis of GA. The as prepared GA is a new exotic form of the highly porous 3D sponge like architecture of graphene nanosheets, which improves the electronic properties in comparison to the parent GO nanosheets and conventional carbon aerogel. Based on GO, resorcinol (R) and formaldehyde (F) as precursors in its synthesis, 3D cross-linked GA is gaining attention in the field of EMI shielding due to its extraordinary mechanical, thermal, electrical and chemical properties. The work described in this communication reignites further research to occur to establish GA as a next-generation effective EMI shielding material.

In the present investigation, GA was prepared using customised sol-gel polymerization chemistry. Initially, GO was synthesized by rigorously following the known synthesis protocol prescribed. It is regularly prepared in our laboratory. ${ }^{27}$ Briefly speaking, first of all graphite oxide is prepared by following the Staudenmaier method. The graphite oxide was then repeatedly washed and dried. It was then subjected to thermal exfoliation by placing it in a pre-heated furnace (at $1050{ }^{\circ} \mathrm{C}$ ) under Ar atmosphere. The resulting material, as verified by Raman and FTIR (Fig. S1 and S2 $\dagger$ ), was GO. After that, GO was thoroughly dispersed in deionised water using ultrasonication for 24 hours. The concentration of GO in water was 1 $\mathrm{wt} \%$. Then resorcinol (R, $0.625 \mathrm{~g})$ and formaldehyde (F, $0.9 \mathrm{~g}$ ) with sodium carbonate as a catalyst $(\mathrm{C}, 3 \mathrm{mg}$ ) were added to the GO suspension, followed by magnetic stirring for 1.5 hours. The resorcinol and formaldehyde (RF solids) were $4 \mathrm{wt} \%$ of the reactant concentration in the starting mixture. The molar ratio of $\mathrm{R}: \mathrm{C}$ and $\mathrm{R}: \mathrm{F}$ was $200: 1$ and $1: 2$, respectively. The final reaction mixture was poured into glass vials and cured in an oven at $85{ }^{\circ} \mathrm{C}$ ( 3 days) for gelation. Moreover, the wet cross linked gels were exchanged with acetone to extract the water content present in the pores. A supercritical drying process was employed to dry the wet gels using liquid $\mathrm{CO}_{2}$. Finally, the carbonization was carried out at $900{ }^{\circ} \mathrm{C}$ for 3 hours under ambient nitrogen which yielded the graphene aerogel. The details of the experiment performed (Fig. S3 $\dagger$ ) together with the locally fabricated supercritical dryer (Fig. S4 $\dagger$ ) are given in the ESI. $\dagger$
The structural characterization of the sample was done through an X'Pert PRO (PANalytical) X-ray diffractometer equipped with a graphite monochromator utilizing $\mathrm{CuKa}_{1}$ radiation $(\lambda=1.5406 \AA)$. Fig. 1 (a) demonstrates the representative XRD pattern for the GA, which shows a weak peak at $2 \theta=$ $22.30^{\circ}$ this corresponds to the (002) planes\} suggesting a minimum stacking of (002) and larger interlayer spacing of the sheets. Moreover, there is a broad peak extending from $2 \theta=$ 38 to $55^{\circ}$. This comes from the $(100) /(101)$ plane, which is consistent with other reports. ${ }^{26}$ The representative Raman spectrum of GA taken using a Horiba LabRAM HR800 (Argon LASER light of wavelength $514 \mathrm{~nm}$ ) is shown in Fig. 1(b). It has intense D $\left(1336 \mathrm{~cm}^{-1}\right)$ and $\mathrm{G}\left(1582 \mathrm{~cm}^{-1}\right)$ bands with broad 2D features, which are similar to the known Raman peaks for GA. The strong D band in the bulk graphene aerogel spectrum is probably because of the many junctions inbuilt in the $3 \mathrm{D}$ graphene network. ${ }^{28}$ Fourier transform infrared spectroscopy (FTIR) was obtained using a Perkin Elmer FTIR spectrometer (spectrum 100) in transmission mode in the wavenumber range of $500-4000 \mathrm{~cm}^{-1}$. The FTIR spectrum of the graphene aerogel is shown in Fig. 1(c). The broad and strong peak near $3485 \mathrm{~cm}^{-1}$ indicates the stretching vibration of the $\mathrm{O}-\mathrm{H}$ bond. In addition, the peak at $1645 \mathrm{~cm}^{-1}$ can be attributed to the $\mathrm{C}=\mathrm{C}$ stretching vibration. The peak around $1355 \mathrm{~cm}^{-1}$ results from the stretching vibrations of $\mathrm{C}-\mathrm{O}$ in the carboxyl group. The BET

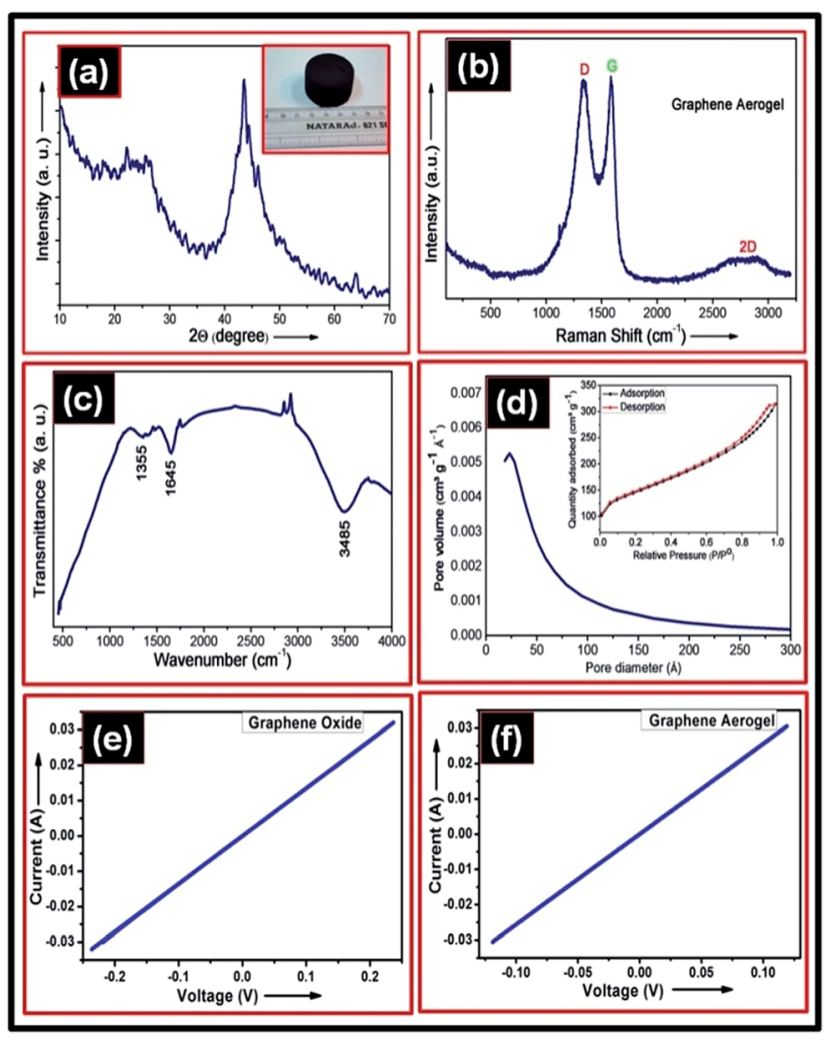

Fig. 1 (a) XRD pattern of GA, inset is the optical image of GA, (b) Raman spectrum of GA, (c) FT-IR spectrum of GA, (d) pore volume versus pore diameter graph at STP, inset is the nitrogen adsorption/ desorption isotherm, and (e and f) $I-V$ curve of GO and GA, respectively. 
surface area was determined by a surface area analyzer (Micrometrics, USA, ASAP 2020 Models). The nitrogen porosimetry data indicates the textural properties of the graphene aerogel. The nitrogen adsorption/desorption isotherm the inset of Fig. 1(d)\}indicates that significant mesoporosity exists, which is consistent with the sheet-like structure observed in the electron micrographs. The actual BET surface area and pore volume of the graphene aerogel obtained is $516 \mathrm{~m}^{2} \mathrm{~g}^{-1}$ and $0.485725 \mathrm{~cm}^{3} \mathrm{~g}^{-1}$, respectively. The pore size distribution $\{$ Fig. $1(\mathrm{~d})\}$ shows that the peak pore diameter is $\sim 2.5 \mathrm{~nm}$, which depends upon the RF polymer content in the initial precursor. The DC conductivity of the GO and GA was determined via the four-point probe system (Keithley 6220 precision current source, Keithley 2182A Nanovoltmeter). Fig. 1(e) and (f) represent the room temperature $I-V$ characteristics of GO and GA, respectively. The conductivities obtained for the GO and GA samples are $85 \mathrm{~S} \mathrm{~m}^{-1}$ and $130 \mathrm{~S} \mathrm{~m}^{-1}$, respectively. Here it is found that GA has a higher conductivity compared to GO. This may be due to the reduction in resistance at the junction between the graphene sheets.

The surface morphological study of the as prepared GA was carried out using scanning electron microscopy (SEM) \{FEI, Quanta 200\}. Fig. 2(a) and (b) demonstrate the representative SEM micrographs of the graphene aerogel at low and high magnification, respectively. It can be clearly seen that Fig. 2(b) exhibits a 3D set of connections of randomly distributed sheetlike structures. These sets of sheets are thin enough to be transparent to the electron beam. The microstructural characterization was performed using an FEI Tecnai-20 $\mathrm{G}^{2}$ acceleration voltage $(200 \mathrm{kV})$ high-resolution transmission electron microscope (HRTEM). The representative TEM and HRTEM micrographs of the graphene aerogel are shown in Fig. 2(c) and (d), at different magnifications. The TEM micrographs reveal a wrinkled paper-like texture to the sheets, which is consistent with previous studies. ${ }^{29,30}$

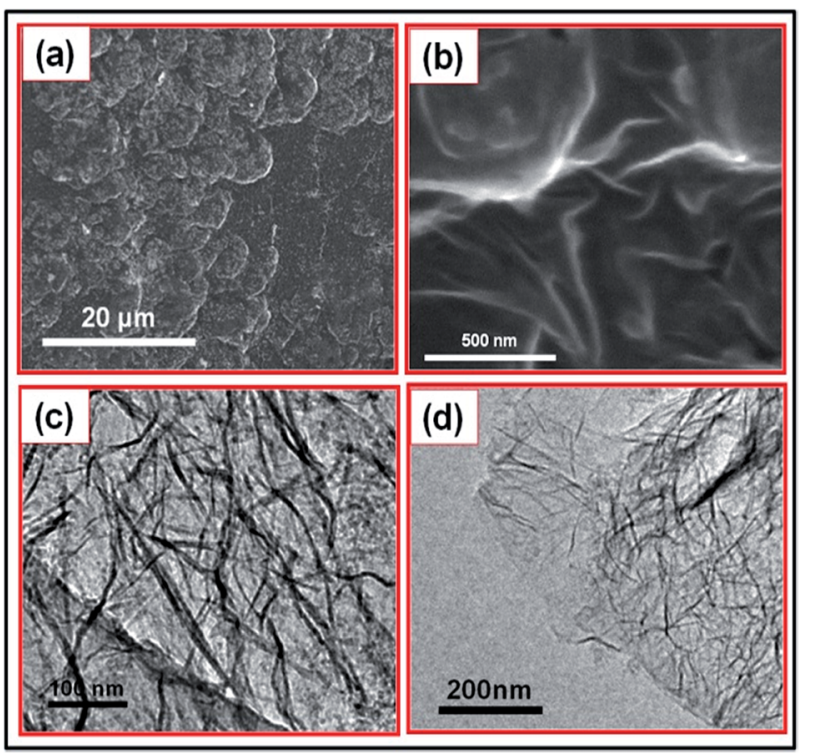

Fig. 2 (a) \& (b) Low and high magnification SEM images and (c) \& (d) TEM images of the as prepared GA.
EMI shielding measurements were carried out on an Agilent E8362B vector network analyzer in the microwave range of 12.418.0 GHz. For this, the powder sample was pressed into a $\sim 2$ $\mathrm{mm}$ thick rectangular shaped pellet with a dimension to fit the waveguide dimensions. The EMI shielding effectiveness (SE) of a material is defined as the ratio of transmitted power to incident power and is given by $\mathrm{SE}_{\mathrm{T}}(\mathrm{dB})=-10 \log \left\{P_{\mathrm{T}} / P_{\mathrm{I}}\right\}$ where $P_{\mathrm{I}}$ and $P_{\mathrm{T}}$ are the incident and transmitted power of the EM waves, respectively. When an EM wave is incident on the shield it is partly absorbed, reflected and transmitted. Therefore, the total shielding effectiveness is the sum of the shielding effectiveness due to reflection $\left(\mathrm{SE}_{\mathrm{R}}\right)$ and absorption $\left(\mathrm{SE}_{\mathrm{A}}\right)$ and is given by the expression $\mathrm{SE}_{\mathrm{T}}=\mathrm{SE}_{\mathrm{A}}+\mathrm{SE}_{\mathrm{R}}$. The scattering parameters $S_{11}$ (or $S_{22}$ ) and $S_{21}$ (or $S_{12}$ ) of a two port network analyzer can be related with the reflectance and transmittance as, $T=\left|S_{21}\right|^{2}=\left|S_{12}\right|^{2}$, $R=\left|S_{11}\right|^{2}=\left|S_{22}\right|^{2}$.

The absorbance $(A)$ can be written as $A=(1-R-T)$. Here, it should be noted that the absorption coefficient is given with respect to the power of the incident $\mathrm{EM}$ wave. The relative intensity of the effectively incident EM wave inside the material after first reflection is based upon the quantity $(1-R)$. Therefore, the effective absorbance ( $A_{\text {eff }}$ ) can be described as $A_{\text {eff }}=$ $(1-R-T) /(1-R)$ with respect to the power of the effectively incident EM wave inside the shielding material. Therefore, it is convenient to express the reflection and effective absorption losses in the form of $-10 \log (1-R)$ and $-10 \log \left(1-A_{\text {eff }}\right)$ respectively, which give $\mathrm{SE}_{\mathrm{R}}$ and $\mathrm{SE}_{\mathrm{A}}$ as $\mathrm{SE}_{\mathrm{R}}=-10 \log (1-R)$ and $\mathrm{SE}_{\mathrm{A}}=-10 \log \left(1-A_{\text {eff }}\right)=-10 \log (T / 1-R)$.

To show the excellent EM wave attenuation performances of GA with the changing frequency $T$ (transmittance coefficient), $R$ (reflection coefficient) and $A$ (absorption coefficient) values were calculated and are shown in Fig. 3(a), (c) and (e). According to the plots exhibited in Fig. 3(e), the $A$ values are in the range of 0.5 to 0.6 while the $R$ values lie between 0.30 and 0.45 . The $T$ values are significantly small and lie within the range 0.008-0.024. Interestingly, $T$ decreases with increasing frequency. Such small values show that the synthesized GA has potential shielding properties. In other words, due to the higher $A$ and $R$ coefficient, more EM waves are consumed by GA. This leads to a significant decrease of the $T$ values. Fig. 3(b), (d) and (f) show the variation of $\mathrm{SE}_{\mathrm{T}}, \mathrm{SE}_{\mathrm{R}}$, and $\mathrm{SE}_{\mathrm{A}}$ in the frequency range of 12.4-18.0 GHz. From the experimental measurement of GA, the shielding effectiveness due to absorption $\left(\mathrm{SE}_{\mathrm{A}}\right)$ has been found to vary from $\sim 14.5$ to 18.0 $\mathrm{dB}$ while $\mathrm{SE}_{\mathrm{R}}$ varies from $\sim 1.9$ to $2.3 \mathrm{~dB}$. Thus, the total $\mathrm{SE}_{\mathrm{T}}$ achieved for the GA is $\sim 20.0 \mathrm{~dB}$, which is much higher than the pristine graphene oxide (GO) nanosheets. It is very interesting to note that GA is a better selection over the conventional GO not only in the sense that it shows higher total $\mathrm{SE}$, but also $\mathrm{SE}_{\mathrm{R}}$ is much less in GA as compared to GO. It has been observed that for GA, the shielding effectiveness (SE) is mainly dominated by absorption while the shielding effectiveness due to reflection $\left(\mathrm{SE}_{\mathrm{R}}\right)$ is almost constant, and it contributes negligibly.

In order to understand the contribution of the multiple reflections that are produced by the coupling of the reflected radiation on the first incidence plane and the reflection in the final plane of the material, the skin depth of the graphene aerogel shield has been evaluated using the relation 


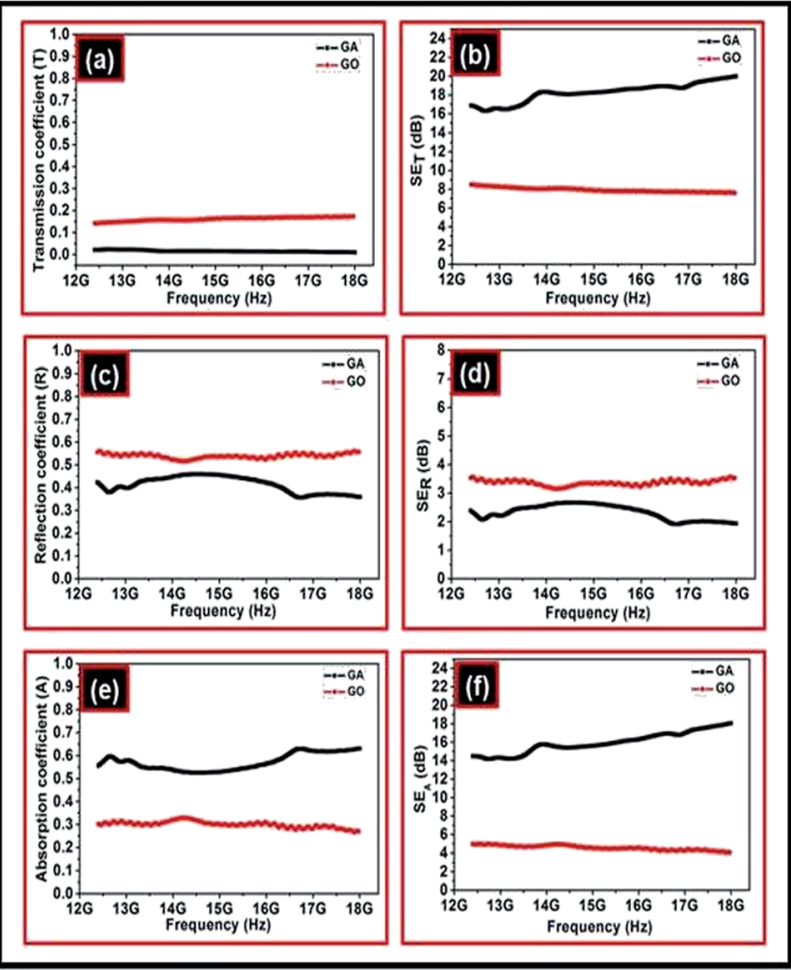

Fig. 3 (a) Transmission (T), (c) refection (R), and (e) absorption (A) coefficients and the shielding effectiveness (b) $S E_{T}$, (d) $S E_{R}$, and (f) $S E_{A}$ of the as synthesized $\mathrm{GA}$ as well as the pristine $\mathrm{GO}$ in the frequency range of $12.4-18.0 \mathrm{GHz}$.

$$
\delta=\sqrt{2 / \omega \mu \sigma_{\mathrm{ac}}}
$$

and its variation with frequency has been shown in Fig. 4(a). The average skin depth of the graphene aerogel is found to be $\sim 0.8 \mathrm{~mm}$, which is smaller than the thickness of the shield (2 $\mathrm{mm})$. According to SchelKunoff's theory, multiple reflections can be neglected when the thickness of the shield is greater

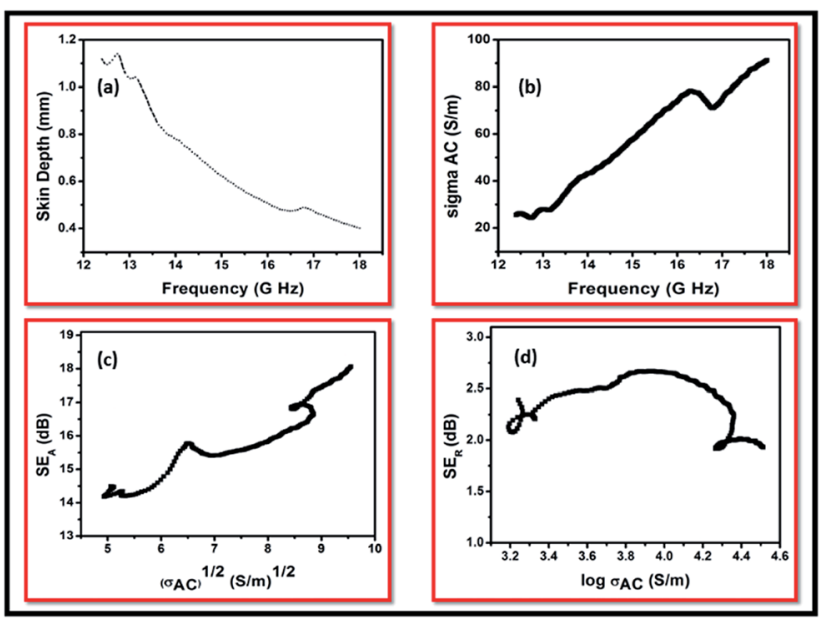

Fig. 4 (a) Plot of the skin depth vs. frequency, (b) variation of $\sigma_{\mathrm{ac}}$ with the increase in frequency, (c) dependence of $\mathrm{SE}_{\mathrm{A}}$ as a function of $\left(\sigma_{\mathrm{ac}}\right)^{1 / 2}$, and (d) variation of $\mathrm{SE}_{\mathrm{R}}$ as a function of $\log \sigma_{\mathrm{ac}}$ for the graphene aerogel. than the skin depth. Therefore, the term $\mathrm{SE}_{\mathrm{M}}$ can be neglected. According to EM theory, for electrically thick samples $(t>\delta)$, frequency $(\omega), \mathrm{SE}_{\mathrm{R}}$ and $\mathrm{SE}_{\mathrm{A}}$ can be expressed in terms of the conductivity $\left(\sigma_{\mathrm{ac}}\right)$, real permeability $\left(\mu^{\prime}\right)$, skin depth $(\delta)$ and thickness $(t)$ of the shield material as:

$$
\mathrm{SE}_{\mathrm{R}}(\mathrm{dB})=10 \log \left\{\sigma_{\mathrm{ac}} / 16 \omega \varepsilon_{0} \mu^{\prime}\right\}
$$

$$
\mathrm{SE}_{\mathrm{A}}(\mathrm{dB})=20\{t / \delta\} \log e=20 d \sqrt{\mu \omega \sigma_{\mathrm{ac}} / 2 \log e}=8.68\{t / \delta\}
$$

$\sigma_{\mathrm{ac}}$ can be related to the imaginary permittivity $\left(\varepsilon^{\prime \prime}\right)$ as $\sigma_{\mathrm{ac}}=$ $\omega \varepsilon_{0} \varepsilon^{\prime \prime}$ and its behavior has been shown in Fig. 4(b). The variation of the dielectric attributes $\left(\varepsilon^{\prime}, \varepsilon^{\prime \prime}\right)$ is shown in the ESI (S5). $\dagger$ To relate $\sigma_{\mathrm{ac}}$ with the shielding parameter of the material, $\mathrm{SE}_{\mathrm{A}}$ has been plotted against $\left(\sigma_{\mathrm{ac}}\right)^{1 / 2}$ (Fig. $4(\mathrm{c})$ ). From the above equation, it is seen that a better $\mathrm{SE}_{\mathrm{A}}$ can be achieved from moderate conducting materials. The dependence of $\mathrm{SE}_{\mathrm{R}}$ as a function of $\log \sigma_{\mathrm{ac}}$ is shown in Fig. 4(d). Therefore, to minimize SE due to reflection, a moderate value of conductivity $\left(\sigma_{\mathrm{ac}}\right)$ is required.

The plausible mechanism of EMI shielding through GA is shown in Fig. 5. The proposed reason behind the observed SE is the highly porous, light weight 3D sponge like structure of the graphene aerogel, which provides the high surface area. The defects on the boundary of GA hinder the path of the electrons, which contributes to dielectric losses and dissipates microwave energy. The dielectric losses occurring in GA are the result of the electronic and space charge polarization. The contribution to the space-charge polarization appears to be due to the space charge accumulating at the interface of the various layers leading to field distortion.

In conclusion, we demonstrate the microwave shielding properties of light weight 3D GA derived from graphene oxide (GO). The structural, microstructural and morphological characteristics of this nanostructure have been elucidated by XRD, SEM and TEM/HRTEM. The microstructural results clearly reveal that the GA 3D architecture is composed of high-quality graphene layers with a very high surface area of $\sim 516 \mathrm{~m}^{2} \mathrm{~g}^{-1}$. This engineered GA exhibits an enhanced shielding

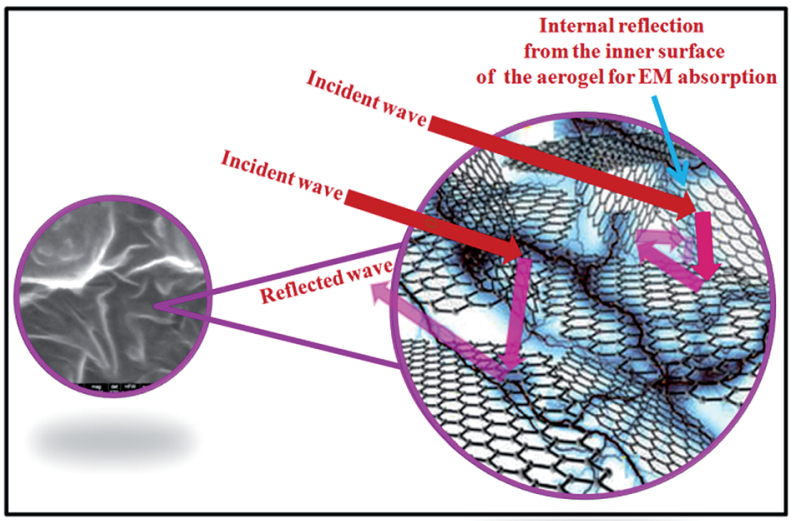

Fig. 5 Proposed mechanism of the EMI shielding process through GA. 
effectiveness $(\sim 20.0 \mathrm{~dB}$ at $0.2 \mathrm{~g}$ in the frequency range 12.4 to 18.0 GHz) as compared to the conventional pristine GO. It has been found that GA improves the dielectric loss of GO, which helps to increase the microwave shielding effectiveness in terms of its high microporosity (pore diameter $\sim 2.5 \mathrm{~nm}$ ), higher specific surface area, lower density and higher electrical conductivity. Moreover, the high microporosity of GA provides a better polarity along with a good absorption capacity. Thus, the present result of the EMI shielding of GA offers a new analogue of graphene in the form of GA. This could serve as a new material for next-generation EMI shielding applications.

\section{Acknowledgements}

The authors acknowledge MNRE (Mission mode project on Hydrogen Storage), DST, UGC and HWB (DAE), and CMPDI (Ministry of coal) India for financial support. We would also like to thank Prof. M. A. Worsley (Lawrence Livermore National Laboratory, USA) and Prof. A. S. K. Sinha (IIT, BHU) for helpful discussions.

\section{Notes and references}

1 F. Qin and C. Brosseau, J. Appl. Phys., 2012, 111, 061301.

2 C. Y. Lee, H. G. Song, K. S. Jang, E. J. Oh, A. J. Epstein and J. Joo, Synth. Met., 1999, 102, 1346.

3 B. R. Kim, H. K. Lee, S. H. Park and H. K. Kim, Thin Solid Films, 2011, 519, 3492.

4 E. Ma, J. Li, N. Zhao, E. Liu, C. He and C. Shi, Mater. Lett., 2013, 91, 209.

5 Y. Yang, M. C. Gupta, K. L. Dudley and R. W. Lawrence, Nano Lett., 2005, 5, 2131.

6 G. S. Wang, X. J. Zhang, Y. Z. Wei, S. He, L. Guo and M. S. Cao, J. Mater. Chem. A, 2013, 1, 7031.

7 B. Wen, M. Cao, Z. Hou, W. Song, L. Zhang, M. Lu, H. Jin, X. Fang, W. Wang and J. Yuan, Carbon, 2013, 65, 124.

8 Y. Qing, X. Wang, Y. Zhou, Z. Huang, F. Luo and W. Zhou, Compos. Sci. Technol., 2014, 102, 161.

9 W. Song, M. Cao, L. Fan, M. Lu, Y. Li, C. Wang and H. Ju, Carbon, 2014, 77, 130.

10 Y. Huang, Y. Wang, Z. Li, Z. Yang, C. Shen and C. He, J. Phys. Chem. C, 2014, 118, 26027.

11 D. Micheli, A. Vricella, R. Pastore and M. Marchetti, Carbon, 2014, 77, 756.

12 M. Cao, W. Song, Z. Hou, B. Wen and J. Yuan, Carbon, 2010, 48, 788.
13 B. Wu, H. M. Tuncer, A. Katsounaros, W. Wu, M. T. Cole, K. Ying, L. Zhang, W. I. Milne and Y. Hao, Carbon, 2014, 77, 814.

14 P. Tripathi, C. R. Prakash Patel, A. Dixit, A. P. Singh, P. Kumar, M. A. Shaz, R. Srivastava, G. Gupta, S. K. Dhawan, B. K. Gupta and O. N. Srivastava, RSC Adv., 2015, 5, 19074.

15 X. J. Zhang, G. S. Wang, W. Q. Cao, Y. Z. Wei, M. S. Cao and L. Guo, RSC Adv., 2014, 4, 19594.

16 D. Chen, G. S. Wang, S. He, J. Liu, L. Guo and M. S. Cao, J. Mater. Chem. A, 2013, 1, 5996.

17 M. Zong, Y. Huang, H. Wu, Y. Zhao, Q. Wang and X. Sun, Mater. Lett., 2014, 114, 52.

18 P. B. Liu, Y. Huang and X. Sun, ACS Appl. Mater. Interfaces, 2013, 5, 12355.

19 Y. Chen, G. Xiao, T. Wang, Q. Ouyang, L. Qi, Y. Ma, P. Gao, C. Zhu, M. Cao and H. Jin, J. Phys. Chem. C, 2011, 115, 13603.

20 Y. Zhan, F. Meng, Y. Lei, R. Zhao, J. Zhong and X. Liu, Mater. Lett., 2011, 65, 1737.

21 Y. Gao and Z. Tang, Small, 2011, 7, 2133.

22 Q. L. Liu, B. Cao, C. L. Feng, W. Zhang, S. M. Zhu and D. Zhang, Compos. Sci. Technol., 2012, 72, 1632.

23 F. Moglie, D. Micheli, S. Laurenzi, M. Marchetti and V. M. Primiani, Carbon, 2012, 50, 1972.

24 Z. Fang, C. Li, H. Sun, H. Zhang and J. Zhang, Carbon, 2007, 45, 2873.

25 H. B. Zhang, Q. Yan, W. G. Zheng, Z. X. He and Z. Z. Yu, ACS Appl. Mater. Interfaces, 2011, 3, 918.

26 M. A. Worsley, T. T. Pham, A. M. Yan, S. J. Shin, J. R. I. Lee, M. Bagge-Hansen, W. Mickelson and A. Zettl, ACS Nano, 2014, 8, 11013.

27 M. S. L. Hudson, H. Raghubanshi, S. Awasthi, T. Sadhasivam, A. Bhatnager, S. Simizu, S. G. Sankar and O. N. Srivastava, Int. J. Hydrogen Energy, 2014, 39(16), 83118320.

28 P. J. Pauzauskie, M. A. Worsley, T. F. Baumann, J. H. Satcher and J. Biener, 2012, US pat., 2012/0034442 A1.

29 M. J. McAllister, J. L. Li, D. H. Adamson, H. C. Schniepp, A. A. Abdala, J. Liu, M. Herrera-Alonso, D. L. Milius, R. Car, R. K. Prud'homme and I. A. Aksay, Chem. Mater., 2007, 19, 4396.

30 M. A. Worsley, P. J. Pauzauskie, T. Y. Olson, J. Biener, J. H. Satcher and T. F. Baumann, J. Am. Chem. Soc., 2010, 132, 14067. 